\title{
Les lois Gamma restreintes, leurs variantes, leurs moments et quelques bribes de leurs distributions Time-constrained Gamma variables, their variants, moments and distribution
}

\author{
Louis Laurencelle $\mathrm{a}^{\mathrm{a},}$ \\ ${ }^{\mathrm{a}}$ Université du Québec à Trois-Rivières
}

\begin{abstract}
The Gamma (or Erlang) variable with integer parameter $k$ can be seen as a global duration obtained through summing $k$ successive individual delays, each one following a standard exponential distribution. What does happen to the Gamma variable if an upper bound is placed either on the individual delays or on their combined duration while the summing of delays continues until the chosen criterion is met? We devised and studied seven likely variants of the constrained Gamma variable, for some of which statistical moments and probability distribution are furnished.
\end{abstract} Acting Editor $\square$ Denis Cousineau (Uni-

versité d’Ottawa)

Keywords $\square$ Constrained Gamma distributions, Cumulative triggering function.

louis.laurencelle@gmail.com

$L L: 0000-0003-3448-2872$

10.20982/tqmp.12.2.p123

\section{Introduction}

Pour décrire et modéliser le comportement statistique des événements accidentels, le processus de Poisson (FELLer, 1968; Olkin, Gleser, \& Derman, 1980), que nous nommerons aussi processus exponentiel, sert souvent de référence. Sommairement et idéalement, l'événement modélisé a une propension de se produire qui ne varie ni dans l'espace ni dans le temps. Par exemple, le nombre d'événements enregistrés tendra à croître en relation proportionnelle avec la durée d'observation, et le délai avant que se produisent tant d'événements successifs du même phénomène croîtra de même avec le nombre d'événements. La chute de la foudre dans le parc du voisinage, les erreurs de typographie par page de texte, la désintégration par seconde d'un matériau radioactif sont autant d'exemples qu'un processus de Poisson permet d'approximer.

Pour un phénomène conforme au processus de Poisson, le nombre $k$ d'événements advenus dans une période donnée suit la loi de Poisson, avec fonction de masse :

$$
p(k)=e^{-\mu} \mu^{k} / k !
$$

Observant le processus de Poisson d'une autre manière, le temps $t$ qu'il faudra attendre pour enregistrer la $k^{\text {ième }}$ occurrence successive du phénomène suit la loi Gamma, de densité :

$$
p(t)=\beta^{k} t^{k-1} e^{-\beta t} / \Gamma(k),
$$

$\beta$ étant un paramètre d'échelle et $\Gamma()$ dénotant la fonction "gamma", où $\Gamma(k)=(k-1)$ !. JoHnson, Kotz, et BALAKRISHNAN (1994) donnent toute la théorie sur la distribution, l'estimation des paramètres et le calcul relatifs à cette variable.

Or, posons une loi Gamma en forme standard, avec $\beta=1$, à paramètre $k$ entier $^{1}$, et de densité de probabilité :

$$
p(t)=t^{k-1} e^{-t} /(k-1) !
$$

La variable $t$, conçue comme le délai d'attente du $k^{\text {ième }}$ événement d'une série d'événements indépendants et successifs, peut être décomposée en $k$ délais individuels $t_{j}$, selon :

$$
t=t_{1}+t_{2}+\cdots+t_{k}
$$

chaque délai $t_{j}$ répond à une loi Gamma de paramètre $k=1 \mathrm{ou}$, équivalemment, à la loi Exponentielle standard,

1. Le modèle Gamma utilisant une valeur $k$ entière est parfois désigné modèle ou loi d’Erlang. 
de densité :

$$
p\left(t_{j}\right)=\exp \left(-t_{j}\right)
$$

Qu'arrivera-t-il à notre variable globale, que nous baptisons dorénavant $T$, si une restriction de durée est imposée à chaque composante $t_{j}$ ou bien à l'ensemble des $k$ composantes de la série? Par exemple, un matériau s'enflamme si une série de 5 impulsions électriques de haut voltage le traverse, chaque impulsion ayant un délai d'apparition moyen de $\frac{1}{2} \mathrm{~s}$, à moins que le délai global des 5 impulsions, trop long, déborde $3 \mathrm{~s}$. Une autre condition pourrait être que chaque impulsion dure en deçà de $1 \mathrm{~s}$, sinon il faut recommencer. Ou encore, pour transmettre le message électrique à la cellule voisine du réseau, la cellule nerveuse doit recevoir 10 ou 15 impulsions synaptiques dans un délai total de $0,1 \mathrm{~s}$. Ainsi, si la condition de succès n'est pas immédiatement atteinte, le processus continue, accumulant le temps perdu, ce jusqu'à satisfaction.

Dans une situation donnée, quelle est la distribution de probabilité de $T$, le délai global requis pour obtenir satisfaction du critère? Quelles sont l'espérance, la variance et les autres propriétés de cette variable? La contrainte de durée maximale imposée pouvant revêtir diverses formes, nous parlons ici de lois Gamma restreintes, au pluriel.

En introduisant la loi de Pascal restreinte en 1983 (voir aussi LAURENCELLE, 2012), Laurencelle proposait déjà cette famille nouvelle de variables statistiques. Les lois Gamma restreintes constituent en effet des généralisations de la loi des succès consécutifs (LAURENCELLE, 1987), d'origine historique lointaine. LAURENCELLE (Mai 1996) semble en avoir posé les premières assises.

Après avoir fixé les principaux éléments de notation et de vocabulaire, nous aborderons à tour de rôle sept réalisations spécifiques que nous jugeons intéressantes dans la famille des lois Gamma restreintes, ces variantes être campées en deux groupes, soit celui des lois dites individuelles et celui des lois totales, chacune étant considérée avec ou sans réamorçage précoce.

\section{Notation et vocabulaire}

Simplifications initiales. La loi Gamma générale (JoHNson et al., 1994, p. 337, éq. 17.1) comporte un paramètre d'origine, un paramètre d'échelle et le nombre $k$ qui détermine l'ordre de la distribution. Nous posons d'emblée l'origine à 0 et le paramètre d'échelle à 1 pour retrouver la loi Gamma standard (3) et ses composantes Exponentielles standard (5). La plupart des résultats et conclusions présentés plus bas ne perdent pas leur généralité sous ces simplifications. Rappelons que nous restreignons ici le paramètre $k$ à des entiers naturels.

La loi Gamma régulière en forme standard a pour den- sité $g(t)$ et fonction de répartition $G(t)$ :

$$
\begin{aligned}
g(t) & =e^{-t} \frac{t^{k-1}}{(k-1) !} \\
G(t) & =e^{-t} \sum_{j=0}^{\infty} \frac{t^{k+j}}{(k+j) !}
\end{aligned}
$$

pour $t \geq 0$. Ses moments principaux sont :

$$
\begin{aligned}
\mu & =\sigma^{2}=k, \\
\gamma_{1} & =2 / \sqrt{k}, \\
\gamma_{2} & =6 / k .
\end{aligned}
$$

On peut scinder l'axe de la variable en deux segments, le premier (Court) limitant supérieurement la variable $t$ à la valeur $L$, l'autre (Long) l'amorçant à cette valeur pour la porter à l'infini, ce qui donne lieu à deux variables distinctes. Les fonctions génératrices suivantes :

$$
\begin{aligned}
G C_{n}(k, L) & =\int_{0}^{L} t^{k-1} e^{-t} d t \\
& =\frac{(k-1+n) !}{(k-1) !}\left[1-e^{-L} \sum_{r=0}^{k-1+n} L^{r} / r !\right], \\
G L_{n}(k, L) & =\int_{L}^{\infty} t^{k-1} e^{-t} d t \\
& =\frac{(k-1+n) !}{(k-1) !} \cdot e^{-L} \sum_{r=0}^{k-1+n} L^{r} / r !
\end{aligned}
$$

permettent d'obtenir respectivement les moments d'ordre $n, A_{n}$ et $B_{n}$, de ces deux variables au moyen de :

$$
\begin{aligned}
& A_{n}=G C_{n}(k, L) / G C_{0}(k, L) ; \\
& B_{n}=G L_{n}(k, L) / G L_{0}(k, L),
\end{aligned}
$$

en plus de définir par :

$$
\Pi=\Pi_{k}=G C_{0}(k, L)
$$

la probabilité d'obtenir un $k$-tuplet 'court'. Par exemple, $A_{1}$ dénote l'espérance mathématique (la "moyenne") de durée d'un délai court, c.-à-d. dont la course est bornée par 0 et $L$, tandis que $B_{2}-B_{1}^{2}$ est la variance d'un délai long.

De plus et à toutes fins pratiques, il est utile de caractériser les ingrédients spécifiques associés à la loi exponentielle, c.-à-d. le cas spécial de la loi Gamma pour lequel $k=1$. Comme le résume le Tableau 1 plus loin, nous dénotons par $t_{C}$ et $\pi$ la durée et la probabilité d'un élément court (tel que $t \leq L$ ) et par $a_{1}$ et $a_{2}$ ses deux premiers moments, puis par $t_{L}$ et $\omega$ la durée d'un élément long $(t>L)$ et sa probabilité, et par $b_{1}$ et $b_{2}$ ses moments. Noter que, par exemple, $a_{1}=A_{1}$.

The Quantitative Methods for Psychology 
Tableau 1 Caractérisation de la loi Exponentielle restreinte

\begin{tabular}{lllll}
\hline Élément & $p(t)$ & $E(t)$ & $E\left(t^{2}\right)$ & $\sigma^{2}(t)$ \\
\hline$t_{C}$ & $\pi=1-e^{-L}$ & $a_{1}=[1-\omega(1+L)] / \pi$ & $a_{2}=\left[2-\omega\left(2+2 L+L^{2}\right)\right] / \pi$ & $\sigma^{2}\left(t_{C}\right)=\left[1-\omega\left(2+L^{2}\right)+\omega^{2}\right] / \pi^{2}$ \\
$t_{L}$ & $\omega=e^{-L}$ & $b_{1}=1+L$ & $b_{2}=2+2 L+L^{2}$ & $\sigma^{2}\left(t_{L}\right)=1$ \\
\hline
\end{tabular}

Notons enfin quelques sommes géométriques auxquelles nous devrons occasionnellement recourir :

$$
\begin{aligned}
Q_{1} & =\sum_{r=0}^{k-1} x^{r}=\left(1-x^{k}\right) /(1-x),|x|<1 \\
Q_{2} & =\sum_{r=0}^{k-1} r \cdot x^{r} \\
& =\frac{1}{(1-x)^{2}}\left[x-x^{k}(k-(k-1)) \cdot x\right]
\end{aligned}
$$

et

$$
\begin{aligned}
Q_{3} & =\sum_{r=0}^{k-1} r(r-1) \cdot x^{r} \\
& =\frac{1}{(1-x)^{3}} \times \\
& {\left[2 x^{2}-x^{k} k(k-1)(1-x)^{2}+2 k \cdot x(1-x)+2 x^{2}\right] }
\end{aligned}
$$

Nous présentons maintenant et examinons quelques variantes possibles de la famille de lois que nous avons baptisées lois Gamma restreintes en considération de la restriction de durée qu'on lui impose, la borne (temporelle) $L$ étant appliquée soit à chaque ingrédient (chaque composante Exponentielle de la loi Gamma considérée), soit à une somme d'ingrédients successifs.

\section{Loi GR[ICR] - Gamma à contrainte Individuelle, série Complète avec Reprise}

La série de $k$ événements $\left(t_{j}, t_{j+1}, \cdots, t_{j+k-1}\right)$ est réalisée. À la fin, si tous les $t<L$, le temps accumulé (depuis $j=1$ ) est validé, sinon la série entière est oubliée et le processus recommence à $j=k+1$.

Analyse : Le processus se déroule comme une suite de groupes ou $k$-tuplets, jusqu'à l'obtention d'un $k$-tuplet entièrement formé d'éléments courts, $\left(t_{C_{j}}, t_{C_{j}+1}, \cdots, t_{C_{j+k-1}}\right)$. Le défaut de succès tient à l'obtention d'un $k$-tuplet impropre, comprenant au moins 1 élément long, après l'achèvement duquel $k$-tuplet le processus est relancé.

Chaque élément court ayant pour probabilité $\pi$, la probabilité d'observer un groupe formé de $k$ éléments courts est évidemment $\pi^{k}$ et sa durée moyenne, égale à $k \times a_{1}$. La probabilité d'obtenir un groupe différent est $1-\pi^{k}$; quant à la durée $M$ d'un tel groupe mixte, comportant au moins 1 élément long, elle varie selon le nombre (de 1 à $k$ ) d'éléments longs qu'il contient. En général, le $k$ tuplet contiendra $k-r$ éléments courts et $r$ éléments longs $(r>0)$, et, probabilités mises à part, son moment d'ordre $n$ peut s'exprimer comme :

$$
m_{k, r, n}=E\left\{t_{L(r)}+t_{C(k-r)}\right\}^{n},
$$

de sorte que, par exemple, ses moments 1 et 2 sont :

$$
\begin{gathered}
m_{k, r, 1}=r \times b_{1}+(k-r) \times a_{1}, \\
m_{k, r, 2}=r \times b_{2}+r(r-1) \times b_{1}^{2}+(k-r) \times a_{2}+(k-r)(k-r-1) \times a_{1}^{2} .
\end{gathered}
$$

Ainsi, l'espérance de $M$, un $k$-tuplet comportant au moins 1 élément long, est donnée par :

$$
M=\frac{\sum_{1}^{k}\left(\begin{array}{l}
k \\
r
\end{array}\right) \omega^{r} \pi^{k-r}\left(r \cdot b_{1}+(k-r) \cdot a_{1}\right)}{1-\pi^{k}} .
$$

Le processus de type GR[ICR] imite une loi géométrique, qui guette l'apparition d'un groupe court, apparition retardée par l'apparition possible de groupes mixtes, soit, en calcul conditionnel ${ }^{2}$ :

$$
E(X)=\pi^{k} \times k \times a_{1}+\left(1-\pi^{k}\right)(M+X),
$$

la solution étant :

$$
\begin{aligned}
E(X) & =k \cdot a_{1}+\frac{\left(1-\pi^{k}\right) \cdot M}{\pi^{k}} \\
& =\frac{k}{\pi^{k}} .
\end{aligned}
$$

L'expression du moment 2 à l'origine, $E\left(X^{2}\right)$, n'a pu être réduite. Nous obtenons :

$$
\begin{aligned}
E\left(X^{2}\right) & =k \times a_{2}+k(k-1) a_{1}^{2} \\
& +\sum_{r=1}^{k}\left(\begin{array}{l}
k \\
r
\end{array}\right)(1-\pi)^{r} \pi^{k-r}\left(Z_{1}+Z_{2}+Z_{3}+Z_{4}\right) / \pi^{k}
\end{aligned}
$$

2. Le calcul par conditionnement sur le ou les premiers événements peut s'illustrer simplement par le calcul des moments de la loi géométrique, de fonction de masse $p(n)=(1-\pi)^{n-1} \pi$. Le succès immédiat, pour $n=1$, a pour probabilité $\pi$, alors que son contraire, à probabilité $1-\pi$, ajoute +1 à son espérance, soit $E\left(n^{r}\right)=\pi \times 1^{r}+(1-\pi) \times(1+n)^{r}$. Pour le moment $r=1$, la solution de cette équation donnera rapidement $E(n)=1 / \pi$; ainsi pour les autres moments. 
où :

$$
\begin{aligned}
& Z_{1}=r b_{2}+r(r-1) b_{1}^{2}, \\
& Z_{2}=(k-r) a_{2}+(k-r)(k-r-1) a_{1}^{2}, \\
& Z_{3}=2 r(k-r) a_{1} b 1, \\
& Z_{4}=\left(2 r b_{1}+2(k-r) a_{1}\right) \times E(X) .
\end{aligned}
$$

La "forme" de cette loi, représentant la réalisation éventuelle d'un événement à probabilité donnée (ici, $\pi^{k}$ ), est certes acoquinée à celle de la loi géométrique, laquelle aurait une probabilité de $\pi^{k} / k$, soit l'inverse de $E(X)$. Cependant, le report de l'occurrence de l'événement escompté s’appuie non seulement sur la non-réalisation de celui-ci mais sur la réalisation d'un événement plus long (de grandeur moyenne $M$, indiquée plus haut), de sorte que, par exemple, l'espérance $E\left(X^{2}\right)$ et la variance associée excèdent les valeurs qu'aurait fait anticiper l'espérance $E(X)$.

Les moments d'ordre supérieur peuvent s'obtenir de la même façon. Le tableau 3 (voir en fin de texte) présente quelques valeurs représentatives de cette loi, à toutes fins utiles.

Distribution de probabilité (densité et répartition) : L'étude de la densité et de la fonction de répartition de la loi GR[ICR] dans son cas général n'a pas abouti. Seul le cas le plus simple, celui pour $k=1$, a été résolu et il correspond aussi (trivialement) au cas pareil des lois GR[IT] et GR[TCR] : la solution détaillée apparaît plus bas, pour la loi GR[IT], de même que quelques centiles illustratifs. Le tableau 3 fournit quelques centiles estimés pour deux cas de la loi GR[ICR].

\section{GR[IT] - Gamma à contrainte Individuelle et série Tronquée}

La série est amorcée jusqu'à concurrence soit de $k$ événements courts $t_{j}<L$, soit au premier événement long noté $j^{\prime}$. Dans le premier cas, la série est validée, sinon la durée $j^{\prime}$ est épuisée et le processus 'roule', une nouvelle série étant amorcée à $j=j^{\prime}+1$.

Analyse : Le $k$-tuplet espéré peut se réaliser d'emblée, selon une probabilité $\pi^{k}$. À defaut d'une telle occurrence, un premier temps long peut advenir avec probabilité $1-\pi$, retardant d'un temps l'occurrence attendue selon $X=$ $t_{L}+X$; ou bien 1 temps court suivi d'un long, avec probabilité $(1-\pi) \pi$, selon $X=t_{C}+t_{L}+X$, etc. Par exemple, pour $k=3$, nous avons:

$$
\begin{aligned}
X & =\pi^{3}\left(t_{C}+t_{C}+t_{C}\right)+(1-\pi)\left(t_{L}+X\right) \\
& +(1-\pi) \pi\left(t_{C}+t_{L}+X\right)+(1-\pi) \pi^{2}\left(t_{C}+t_{C}+t_{L}+X\right),
\end{aligned}
$$

dont la solution est :

$$
E(X)=3 a_{2}+\left(1-\pi^{3}\right)\left(b_{1}+X\right)+(1-\pi)\left(\pi+2 \pi^{2}\right) a_{1}
$$

ou, généralement (voir (12) plus haut) :

$$
\begin{aligned}
E(X) & =k a_{1}+(1-\pi)\left(Q_{1} b_{1}+Q_{2} a_{1}\right) / \pi^{k} \\
& =\frac{1-\pi^{k}}{\pi^{k}(1-\pi)},
\end{aligned}
$$

une expression identique à celle l'espérance du nombre d'essais requis pour obtenir $k$ succès consécutifs dans un processus de Bernoulli à probabilité $\pi$ (LAURENCELLE, 1987, 2012). Nous trouvons aussi :

$$
E\left(X^{2}\right)=k \cdot a_{2}+k(k-1) \cdot a_{1}^{2}+\frac{\left(1-\pi^{k}\right)\left[b_{2}+2 b_{1} \cdot E(X)\right]+(1-\pi)\left[Q_{2}\left\{a_{2}+2 a_{1} b_{1}+2 a_{1} E(X)\right\}+Q_{3} a_{1}^{2}\right]}{\pi^{k}} .
$$

Nous ne sommes pas parvenu à réduire cette formule.

\section{Distribution de probabilité (densité et répartition)}

Nous nous sommes attaqué d'abord à la forme la plus simple de la loi GR[IT], soit celle pour laquelle $k=1$. Les réalisations éventuelles de cette loi sont : $t_{C}, t_{L}+t_{C}, t_{L}+$ $t_{L}+t_{C}$, etc. Le temps court déterminant $\left(t_{C}\right)$ peut donc être précédé de zéro, un ou quelques temps longs. La probabilité d'un temps court étant $\pi=1-\exp (-L)$, sa densité $p_{C}\left(t_{C}\right)$ est $e^{-t} / \pi$, tandis que pour un temps long, on a complémentairement $p_{L}\left(t_{L}\right)=p\left(V_{1}\right)=e^{-V} /(1-\pi)=$
$e^{L-V}$. Soit $V_{2}=t_{L 1}+t_{L 2}$, la somme de deux temps longs : $t_{L 1}$ varie de $L$ à $V-L, t_{L 2}$ étant alors égal à $V-t_{L 1}$, d'où :

$$
\begin{aligned}
p\left(V_{2}\right) & =\int_{L}^{\mathrm{V}-L} e^{L-t} \times e^{L-\mathrm{V}+t} d t \\
& =e^{2 L-V}(V-2 L) .
\end{aligned}
$$

De même, pour la somme de $n$ temps longs, la densité s’obtient par :

$$
p\left(V_{n}\right)=\frac{e^{n L-V}(V-n L)^{n-1}}{(n-1) !} ;
$$


la densité relative dans la distribution de $T$, la durée totale écoulée, est donnée en multipliant la densité ci-dessus par la probabilité d'enregistrer $n$ temps longs, soit $(1-\pi)^{n}=$ $e^{-n L}$.

Pour calculer la densité globale $p(T)$, considérons le continuum $T \in[0 . . \infty)$ comme étant découpé en segments de mesure $L$. Dans le segment $[0 . . L)$, seul un temps court est admissible et $T=t_{C}$. Dans $[L . .2 L)$, un temps long est requis, ne débordant pas $2 L$, et est suivi d'un temps court, d'où $T=t_{L}+t_{C}=V_{1}+t_{C}$. En général, dans le segment $[n L . .(n+1) L)$, de 1 seul à un maximum de $n$ temps longs peuvent intervenir, selon leur probabilité propre $(1-\pi)^{n}$, et ils sont suivis d'un temps court. La détermination de chaque densité constitutive et leur somme pondérée permettent d'établir la densité globale de la variable $T$, selon l'expression :

$$
p(T)=\sum_{n=0}^{\lfloor T / L\rfloor}(1-\pi)^{n} p\left(V_{n}\right) .
$$

Dans l'exemple du segment $[2 L . .3 L)$, deux processus sont admissibles, soit $V_{1}+t_{C}$ et $V_{2}+t_{C}$. Dans la composante $V_{1}+t_{C}$, la durée $V_{1}$ a pour bornes de variation $T-L$ et $T$; la borne inférieure $T-L$, plutôt que $L$, est requise pour garder la variable $t_{C}$ sous la barre $L$. La densité $p_{1}(T)$ pour cette composante est alors la convolution $\int_{T-L}^{T} p_{V_{1}}(x) \times p_{C}(T-x) d x$. Pour la composante $V_{2}+t_{C}$, étant donné que par définition $V_{2}>$ $2 L$, ses bornes sont simplement $2 L$ et $T$, d'où $p_{2}(T)=$ $\int_{2 L}^{T} p_{V_{2}}(x) \times p_{C}(T-x) d x$. Dans les deux cas, la variation de $t_{C}$ court de $T-V_{n}$ à $T$. La composition de ces deux densités, selon les probabilités $\pi^{n}=e^{-n L}$, produit :

$$
\begin{aligned}
p(T) & =(1-\pi) p_{1}(T)+(1-\pi)^{2} p_{2}(T) \\
& =e^{-L} \int_{T-L}^{T} p_{V_{1}}(x) \times p_{C}(T-x) d x \\
& +e^{-2 L} \int_{2 L}^{T} p_{V_{2}}(x) \times p_{C}(T-x) d x \\
& =e^{-L} e^{L-T} L+e^{-2 L} e^{2 L-T}\left(\frac{1}{2} T^{2}+2 L-2 L T\right) \\
& =e^{-T}\left(L+\frac{1}{2}\left(T^{2}+2 L-2 L T\right)\right)
\end{aligned}
$$

L'application de cette analyse permet de trouver des expressions de la fonction de densité pour chaque segment temporel, voire une expression générique pour le segment $[n L . .(n+1) L)$. Le tableau 2 plus loin présente ces expressions de même que les fonctions de répartition correspondantes. Nous n'avons pu obtenir de résultats utiles pour $k>1$.

Les moments de la loi GR[IT] sont illustrés au tableau 3. Quant aux centiles, le tableau présente aussi les valeurs exactes pour des lois où $k=1$ ainsi que des estimations Monte Carlo pour $k=4$ et 6 .

\section{GR[ITP] - Gamma à contrainte Individuelle, série} Tronquée avec Plafonnement

Le processus GR[ITP] est le frère du processus GR[IT] ci-dessus, sauf que, en cas d'invalidation par l'occurrence d'un temps long, $t_{j} \geq L$, ce temps $t_{L}$ est tronqué et réduit à $L$, suite à quoi une nouvelle série est amorcée.

Analyse : La durée moyenne de l'élément long, notée $b_{1}$, est ici ramenée à sa borne inférieure $L$. Il suffit donc d'opérer la substitution dans les équations correspondantes associées à la loi GR[IT], d’où, ici :

$$
E(X)=\frac{1-\pi^{k}}{\pi^{k-1}(1-\pi)} .
$$

Quant à $E\left(X^{2}\right)$, sa formule est semblable à celle associée à la loi GR[IT], sauf qu'ici, tel que mentionné, $E\left(t_{L}^{n}\right) \equiv L_{n}$ pour tout $n$ au lieu de $b_{n}$. Des données illustratives apparaissent au tableau 3.

\section{Distribution de probabilité (densité et répartition)}

Tout comme pour la loi GR[IT] traitée précédemment, nous n'avons pu déterminer la densité et la fonction de répartition de la loi GR[ITP] que pour $k=1$. Les réalisations éventuelles du processus dans ce cas sont $T=$ $t_{C}, L+t_{C}, L+L+t_{C}$, etc., ou $T=r L+t_{C}, r \geq 0$. Or, $t_{C}$, qui représente ici, avec $r$, la seule variable proprement dite, a une densité exponentielle, $e^{-t} /\left(1-e^{-L}\right)$, qui ne change pas de forme selon sa position dans l'axe de mesure, donc qui reste indépendante d'un éventuel retard, $r \times L$ : la loi résultante est donc une Exponentielle simple, de densité $e^{-T}$ et fonction de répartition $1-e^{-T}$, caractérisée par $E(T)=\sigma^{2}=1, \gamma_{1}=2$ et $\gamma_{2}=6$.

Le tableau 3 présente les calculs pour la loi GR[ITP] selon $k=1$ et tout $L$ de même que des estimations Monte Carlo pour deux autres cas. Le tableau fournit aussi quelques centiles estimés pour deux cas de la loi GR[ITP].

\section{GR[TCR] - Gamma à contrainte Totale, série Complète avec Reprise}

La série de $k$ événements est réalisée, et seule la somme des temps individuels est considérée. À la fin, si $T_{k}=$ $\sum^{k} t_{j}<L$, la série est validée, sinon une nouvelle série est produite.

Analyse : La somme $T_{k}$ générale est une variable Gamma standard d'ordre $k$, d'espérance et variance égales à $k$ (cf. Eq. 7). Les moments de ses temps court et long, soit $E\left(T_{C}^{n}\right)=A_{n}$ et $E\left(T_{L}^{n}\right)=B_{n}$, sont donnés aux Eqs. (10). Cela étant, le $k$-tuplet représenté par $T_{k}$ est traité ici comme une unité à comportement binaire, son cas favorable $\left(T_{C}\right)$ ayant probabilité $\Pi=\Pi_{k}$ et le processus 
Tableau 2 ॥ Densités $p(T)$ et fonctions de répartition $P(T)$ d'une variable $T$ de loi GR[IT] en fonction du segment temporel de largeur $L$. Noter que, en dernière ligne, $n=\lfloor T / L\rfloor$.

\begin{tabular}{lll}
\hline Intervalle & $p(T)$ & $P(T)$ \\
\hline 0 à $L$ & $e^{-T}$ & $1-e^{-T}$ \\
$L$ à $2 L$ & $e^{-T}(T-L)$ & $1-e^{-T}(1+(T-L))$ \\
$2 L$ à $3 L$ & $e^{-T}(L+(T-2 L) 2 / 2)$ & $1-e^{-T}\left(1+(T-L)+(T-2 L)^{2} / 2\right)$ \\
$3 L$ à $4 L$ & $e^{-T}(L+(2 L T-5 L) 2 / 2+(T-3 L) 3 / 6)$ & $1-e^{-T}\left(1+(T-L)+(T-2 L)^{2} / 2+(T-3 L)^{3} / 6\right)$ \\
$n L$ à $(n+1) L$ & $e^{-T}\left[\sum_{k=1}^{n-1} \frac{\left\{(T-k L)^{k}-(T-(k+1) L)^{k}\right\}}{k !}+\frac{(T-n L)^{n}}{n !}\right]$ & $1-e^{-T} \sum_{k=0}^{n} \frac{(T-k L)^{k}}{k !}$ \\
\hline
\end{tabular}

évoluant selon une simple loi géométrique, à l'instar du processus GR[ICR] traité plus haut. Nous avons donc :

$$
E\left(X^{n}\right)=\Pi \times T_{C}^{n}+(1-\Pi) \times\left(T_{L}+X\right)^{n},
$$

d'où, pour l'espérance :

$$
E(X)=\Pi \times A_{1}+(1-\Pi) \times\left(B_{1}+X\right),
$$

donnant éventuellement :

$$
E(X)=k / \Pi .
$$

L'espérance de $X^{2}$, la variance de $X$ et ses moments supérieurs s'obtiennent pareillement (voir le tableau 3).

Distribution de probabilité (densité et répartition) : Rien de trouvé pour le cas général. Fait exception le cas spécial avec $k=1$, dont la solution correspond (trivialement) au cas similaire de la loi IT (voir plus haut, où le détail de la solution est donné de même que quelques centiles) et de la loi ICR.

Le tableau 3 fournit quelques centiles estimés pour deux cas de la loi GR[TCR].

\section{GR[TCC] - Gamma à contrainte Totale, série Complète avec Continuation}

La série de $k$ événements $\left(t_{j}, t_{j+1}, \cdots, t_{j+k-1}\right)$ est réalisée. À la fin, si $T_{k}=\sum t_{j}<L$, la série est validée, sinon la série se déplace d'un élément, devenant $\left(t_{j+1}, t_{j+2}, \cdots, t_{j+k}\right)$, et le test sur la nouvelle $T_{k}$ est repris, jusqu'à couronnement.

Analyse : La succession éventuelle des essais, sanctionnée par la variable successive $T(j+1)=T(j)-t_{j}+t_{j+k}$, est ainsi marquée d'une dépendance d'ordre $(k-1) / k$. L'analyse ne nous a jusqu'à présent rien donné de concluant concernant la chaîne de probabilités relatives au roulement de la chaîne, ni selon l'accroissement de $L$, ni selon la modification de $k$. Un processus auto-régressif est en jeu.

À défaut de nouvelles pistes d'élucidation et à titre provisoire, le tableau 3 offre des estimations Monte Carlo pour les moments de la variable.

Distribution de probabilité (densité et répartition) : Encore à trouver.
Le tableau 3 fournit quelques centiles estimés pour deux cas de la loi GR[TCC].

\section{GR[TR] - Gamma à contrainte Totale et Réamorçage précoce}

La série est amorcée jusqu'à l'obtention de $k$ événements dont la somme complète est acceptable $\left(\sum^{k} t_{j}<L\right)$ ou jusqu'à l'accumulation d'une somme partielle débordante $\left(\sum^{r} t_{j} \geq L, 1 \leq r \leq k\right)$. Dans le premier cas, la série est validée, sinon une reprise à neuf est amorcée dès l'écoulement de l'événement produisant le $t_{j}$ fatal.

Analyse : Le passage d'un degré $r$ de durée totale courte à l'autre ( $r=1$ à $k$ ) se fait par probabilités $\pi_{1}, \pi_{2 \mid 1}=$ $\pi_{2} / \pi_{1}, \pi_{3 \mid 2}=\pi_{3} / \pi_{2}$, etc. la durée complète aboutissant à $A_{1}$ avec probabilité $\Pi_{k}$. Notons par ailleurs que la première occurrence $\sum^{r} t_{j}>L$ produit un premier élément long $\left(t_{L}\right)$ d'espérance $b_{1}$, quel que soit $r$, plutôt qu'un élément d'ordre $r$ et à espérance $B(r, L)$. Les détours ralentissants ont pour probabilités successives $1-\pi_{1}$ pour $\left(t_{1}\right)_{L}+X=t_{L}+X, \pi_{1} \times\left(1-\pi_{2 \mid 1}\right)$ (pour $\left.\left(t_{1}+t_{2}\right)_{L}+X=t_{L}+X\right)$, etc., ces probabilités correspondant à $1-\pi_{1}, \pi_{1}-\pi_{2}, \cdots, \pi_{k-1}-\pi_{k}$, soit globalement à $1-\pi_{k}=1-\Pi$. La solution générale est donc :

$$
E\left(X^{n}\right)=\Pi \times E\left(T_{C}^{n}\right)+(1-\Pi) \times E\left(t_{L}+X\right)^{n},
$$

d'où :

$$
\begin{aligned}
E(X) & =A_{1}(k, L)+(1-\Pi) b_{1} / \Pi \\
E\left(X^{2}\right) & =A_{2}(k, L)+(1-\Pi)\left(b_{2}+2 b_{1} \times E(X)\right) / \Pi .
\end{aligned}
$$

Les moments d'ordre supérieur s'obtiennent semblablement : voir le tableau 3.

Distribution de probabilité (densité et répartition) : Nous n'avons rien trouvé de substantiel.

Le tableau 3 fournit quelques centiles estimés pour deux cas de la loi GR[TR].

The Quantitative Methods for Psychology 


\section{GR[TRP] - Gamma à contrainte Totale, durée Plafonnée et Réamorçage précoce}

Le processus TRP dérive du processus TR ci-dessus, sauf que, si le total $\sum^{r} t_{j} \rightarrow L$, la série est stoppée dès l'atteinte de $L$, puis reprise à neuf.

Analyse : Le temps long étant ramené à sa borne inférieure, espérance et variance sont réduites. De plus, tel que développé dans LAURENCELLE (1998, p. 20 seq.), la fixation du plafond $L$ à la somme partielle $\sum^{r} t_{j}$ permet de résumer celle-ci à la valeur $L$ et à la probabilité agglutinée $1-\Pi_{k}$. Quant au temps court $T_{C}$ et à sa probabilité $\Pi$, ils sont comme ci-devant.

Ainsi, pour les deux premiers moments, nous avons :

$$
\begin{gathered}
E(X)=\Pi_{k} \times A_{1}+\left(1-\Pi_{k}\right)(L+X) \\
=A_{1}+\left(1-\Pi_{k}\right) L / \Pi_{k} \\
E\left(X^{2}\right)=\Pi_{k} \times A_{2}+\left(1-\Pi_{k}\right)\left(L^{2}+2 L E(X)\right) \\
=A_{2}+\left(1-\Pi_{k}\right)\left(L^{2}+2 L E(X)\right) / \Pi_{k},
\end{gathered}
$$

la variance étant alors :

$$
\begin{aligned}
\operatorname{var}(X) & =E\left(X^{2}\right)-[E(X)]^{2} \\
& =A_{2}-A_{1}^{2}+L^{2}\left(1-\Pi_{k}\right) / \Pi_{k}^{2}
\end{aligned}
$$

Distribution de probabilité (densité et répartition) : Prenons l'exemple d'un processus d'ordre $k=2$. Celui-ci aboutit d'emblée si $T=t_{1}+t_{2} \leq L$, avec probabilité $\Pi_{2}$. Sinon, $t_{1}$ peut déborder et plafonner à $L$, auquel cas la série prend la forme $T=L+T$, avec probabilité $p_{1}$, ou bien $t_{1}+t_{2}$ peut déborder et plafonner à $L$, donnant la série identique $T=L+T$ avec probabilité $p_{2}$. Le réseau des chaînes du processus étant ainsi complété, il est apparent que $\Pi_{2}+p_{1}+p_{2}=1$, d'où $p_{1}+p_{2}=1-\Pi_{2}$, la preuve s'étendant pour tout $k>2$.

Ainsi, à quelque élément $t_{r}$ du $k$-tuplet que se produise la violation $\sum t>L$, la probabilité attachée à cette phase est égale et elle est proportionnelle à $1-\Pi_{k}$. La densité ainsi décrite est multipartite et apparaît comme une répétition de densités Gamma, chaque cycle- $L$ subséquent ayant son amplitude réduite par un facteur $1-\Pi_{k}$ par rapport au cycle antécédent. La densité de $T$, qui s'étend de 0 à $\infty$, s'exprime ainsi par paliers successifs d'étendue $L$, soit $g(T)$ pour $0<T \leq L,\left(1-\Pi_{k}\right) g(T-L)$ pour $L<T \leq 2 L$, $\left(1-\Pi_{k}\right)^{2} g(T-2 L)$ pour $2 L<T \leq 3 L$, etc. Cette structure par étage de la densité donne lieu à la formule suivante :

$$
p(T)=\left(1-\Pi_{k}\right)^{r} \times g(T-r \times L),
$$

où $r=\lfloor T / L\rfloor,(r-1) L<T \leq r L$, et $g(x)$ renvoie à la densité Gamma standard. La fonction de répartition cor- respondante est alors :

$$
\begin{aligned}
P(T) & =\Pi_{k} \sum_{j=1}^{r}\left(1-\Pi_{k}\right)^{j-1}+\left(1-\Pi_{k}\right)^{r} \cdot G(T-r L), \\
& =1-\left(1-\Pi_{k}\right)^{r}+\left(1-\Pi_{k}\right)^{r} \cdot G(T-r L)
\end{aligned}
$$

où $r=\lfloor T / L\rfloor$ et $G(x)$ est la fonction de répartition Gamma standard.

Quant aux moments d'ordre $n, E\left(T^{n}\right)$, ils s'obtiennent par $\sum_{r}\left(1-\Pi_{k}\right)^{r} \times \int g(t) \times(t+r L)^{n} \mathrm{~d} t$ pour $0<t \leq$ $L, T>0$ et $r$ allant de 0 à $\infty$; le lecteur se rappellera que, par exemple, $\int g(t) \mathrm{d} t=\Pi_{k}, \int t g(t) \mathrm{d} t=\Pi_{k} A_{1}$, etc. Ce calcul reproduit celui par conditionnement présenté plus haut. Le tableau 3 présente des valeurs illustratives pour cette loi.

\section{Bilan et perspectives}

Les sept lois, ou variantes, de la famille des lois Gamma restreintes examinées ici n’épuisent pas le domaine des variantes possibles : citons par exemple un processus dans lequel chaque composante devrait respecter une contrainte $L_{I}$ alors que le total serait assujetti à une autre, $L_{T}$, ou encore un dans lequel seules $r$ parmi les $k$ composantes du $k$-tuplet seraient soumises à la contrainte individuelle, etc. Notre intention ici n'était pas d'établir un répertoire exhaustif des éléments de cette famille. Nous nous sommes limité à illustrer la richesse de cette idée et avons tenté d'en proposer, bien maladroitement, certaines solutions.

Le lecteur constatera facilement lui-même le travail qu'il reste à accomplir pour que, déjà, les variantes examinées ici reçoivent le traitement complet que chacune mérite. Il restera aussi à s'intéresser au problème de l'estimation des paramètres et aux approximations possibles des distributions.

\section{Note de l'auteur}

Une première version, incomplète, de cet article a paru en 1998, dans Lettres Statistiques (LAURENCELLE, 1998).

\section{Références}

Feller, W. (1968). An introduction to probability theory and its applications (2 vol.) (3e édition). Wiley : New York.

Johnson, N. L., Kotz, S., \& Balakrishnan, N. (1994). Continous univariate distributions (2 vol.) (2e édition). Wiley : New York.

Laurencelle, L. (1983). La loi de pascal restreinte. Lettres statistiques, 1, 25.

Laurencelle, L. (1987). La loi des succès consécutifs dans un processus de bernouilli. Lettres statistiques, 8, 23-47.

Laurencelle, L. (1993). La loi uniforme : propriétés et applications. Lettres statistiques, 9, 1-23.

The Quantitative Methods for Psychology 
Tableau 3 a Tableau numérique illustratif

\begin{tabular}{|c|c|c|c|c|c|c|c|c|c|c|c|c|c|}
\hline$k$ & $L$ & $E(X)$ & $E\left(X^{2}\right)$ & $\sigma^{2}$ & $\approx \gamma_{1}$ & $\approx \gamma_{2}$ & $C_{05}$ & $C_{10}$ & $C_{25}$ & $C_{50}$ & $C_{75}$ & $C_{90}$ & $C_{95}$ \\
\hline \multicolumn{14}{|c|}{ Loi GR[ICR] } \\
\hline 4 & 1 & 25,053 & 1296,792 & 669,138 & 2,0 & 6,1 & 1,38 & 1,85 & 6,50 & 17,0 & 35,0 & 58,7 & 76,7 \\
\hline 6 & 1,5 & 27,294 & 1494,524 & 749,581 & 2,0 & 6,1 & 2,63 & 3,26 & 7,92 & 18,8 & 37,8 & 63,0 & 82,0 \\
\hline 4 & 1 & 14,307 & 401,613 & 196,922 & 2,0 & 6,1 & 1,373 & 1,837 & 4,25 & 9,95 & 19,7 & 32,6 & 42,4 \\
\hline 6 & 1,5 & 15,905 & 461,559 & 208,581 & 2,0 & 6,1 & 2,63 & 3,26 & 5,46 & 11,41 & 21,46 & 34,7 & 44,8 \\
\hline 1 & 1 & 1,582 & 6,847 & 4,344 & 2,5 & 8,7 & 0,051 & 0,105 & 0,288 & 0,693 & 2,165 & 4,282 & 5,883 \\
\hline 1 & 2 & 1,157 & 3,399 & 2,062 & 2,9 & 11,6 & 0,051 & 0,105 & 0,288 & 0,693 & 1,386 & 2,991 & 4,145 \\
\hline \multicolumn{14}{|c|}{ Loi GR[ITP] } \\
\hline 4 & 1 & 9,044 & 148,142 & 66,352 & 2,0 & 6,0 & 1,37 & 1,81 & 2,24 & 6,54 & 12,19 & 19,6 & 25,3 \\
\hline 6 & 1,5 & 12,356 & 260,220 & 107,542 & 2,0 & 6,0 & 2,63 & 3,23 & 4,92 & 9,16 & 16,35 & 25,9 & 33,1 \\
\hline 4 & 2 & 27,996 & 1625,018 & 841,231 & 2,0 & 6,0 & 1,366 & 1,744 & 7,23 & 19,0 & 39,1 & 65,7 & 86,0 \\
\hline 6 & 3 & 71,498 & 10381,943 & 5269,920 & 2,0 & 6,0 & 2,614 & 6,76 & 19,7 & 49,2 & 99,5 & 166,1 & 216,4 \\
\hline \multicolumn{14}{|c|}{ Loi GR[TCC] } \\
\hline 4 & 2 & 15,05 & 449,3 & 222,8 & 2,0 & 6,1 & 1,366 & 1,744 & 4,35 & 10,4 & 20,8 & 34,5 & 44,9 \\
\hline 6 & 3 & 30,63 & 1827 & 888 & 2,0 & 6,0 & 2,614 & 3,84 & 9,3 & 21,4 & 42,1 & 69,4 & 90,1 \\
\hline \multicolumn{14}{|c|}{ Loi GR[TR] } \\
\hline 4 & 2 & 19,471 & 763,163 & 384,034 & 2,0 & 6,0 & 1,366 & 1,745 & 5,46 & 13,42 & 27,03 & 45,01 & 58,63 \\
\hline 6 & 3 & 46,061 & 4214,137 & 2092,483 & 2,0 & 6,0 & 2,613 & 5,61 & 13,4 & 32,0 & 63,7 & 105,6 & 137,3 \\
\hline \multicolumn{14}{|c|}{ Loi GR[TRP] } \\
\hline 4 & 2 & 13,472 & 349,596 & 168,096 & 2,0 & 6,0 & 1,366 & 1,745 & 3,898 & 9,562 & 17,994 & 29,952 & 39,489 \\
\hline 6 & 3 & 35,145 & 2406,149 & 1170,977 & 2,0 & 6,0 & 2,613 & 5,027 & 11,190 & 23,925 & 47,844 & 80,168 & 103,971 \\
\hline
\end{tabular}

Note. Les moments de forme, $\gamma_{1}$ (asymétrie) $\gamma_{2}$ (aplatissement) et les centiles sont estimés par Monte Carlo.

Laurencelle, L. (1998). Les lois gamma restreintes. Lettres Statistiques, 10, 67-84.

Laurencelle, L. (2005). Le calcul des moments et de la distribution de probabilité de la loi de pascal restreinte par la chaine de Markov. Lettres Statistiques, 12, 6580.

Laurencelle, L. (2012). La loi de pascal restreinte et ses cas particuliers [constrained pascal distribution and its special cases]. The Quantitative Methods for Psychology, 8, 35-51.

Laurencelle, L. (Mai 1996). La loi gamma restreinte et ses variantes. In Communication au 64e congrès annuel de l'ACFAS.

Olkin, I., Gleser, L. J., \& Derman, C. (1980). Probability models and applications. Macmillan : New York.

\section{Citation}

Laurencelle, L. (2016). Les lois Gamma restreintes, leurs variantes, leurs moments et quelques bribes de leurs distributions time-constrained gamma variables, their variants, moments and distribution. The Quantitative Methods for Psychology, 12(2), 123-130. doi :10.20982/tqmp.12.2.p123

Copyright ( $)$ 2016, Laurencelle. This is an open-access article distributed under the terms of the Creative Commons Attribution License (CC BY). The use, distribution or reproduction in other forums is permitted, provided the original author(s) or licensor are credited and that the original publication in this journal is cited, in accordance with accepted academic practice. No use, distribution or reproduction is permitted which does not comply with these terms.

Received: 31/01/2016 Accepted: 10/02/2016 